\title{
HADRONIC CONTRIBUTIONS TO THE MUON $g-2$ AND LOW-ENERGY QCD
}

\author{
Eduardo de RAFAEL
}

\begin{abstract}
The contributions to the muon anomalous magnetic moment from hadronic vacuum polarization and from hadronic light-by-light scattering are reexamined within the frame work of chiral perturbation theory; the $1 / N_{c}$-expansion; and the extended Nambu Jona-Lasinio model of low-energy QCD.
\end{abstract}

October 1993 
The anomalous magnetic moment of the muon is known to an accuracy of less than 10ppm. The latest result from the combined $\mu^{+}$and $\mu^{-}$measurements performed at CERN is $[1]$

$$
a_{\mu} \equiv \frac{1}{2}\left(g_{\mu}-2\right)=11659230(85) \times 10^{-10} .
$$

The standard model prediction quoted in the most recent review article on the subject [2] is

$$
a_{\mu}^{S M}=11659192.8(17.6) \times 10^{-10} .
$$

This includes the pure QED contributions from leptons to four-loops in perturbation theory, as well as the lowest order weak interaction contribution

$$
a_{\mu}^{\text {weak }}=19.5(1.0) \times 10^{-10} ;
$$

and the contributions from hadronic vacuum polarization (Figs. 1a and 1b), and from the hadronic light-by-light scattering (Fig. 2). These hadronic contributions amount to a total

$$
a_{\mu}^{H}=702.7(17.5) \times 10^{-10} .
$$

The agreement between the standard model prediction and the present experimental value is rather good.

A new high-precision experiment to measure $a_{\mu}$ with an expected error of $\pm 4 \times 10^{-10}$ is underway at BNL. To confront usefully theory with the new experiment will require a better determination of the hadronic contributions. A substantial improvement in the accuracy of the contribution from the hadronic vacuum polarization is possible. It requires, essentially, a more precise determination of the low energy tail of the total $e^{+} e^{-} \rightarrow$ hadrons cross-section. The VEPP-2M facility currently in operation, and DA $\Phi$ NE in the near future, can attain that precision. By contrast, the contribution to $a_{\mu}$ from the hadronic light-by-light scattering, which we shall denote $a_{\mu}($ had 2$)$, cannot be expressed as a convolution of experimentally accessible observables. Here, we have to resort to theoretical wisdom The result quoted in [2], based on the estimates made by Kinoshita, Nižić and Okamoto (KNO) [3], is

$$
a_{\mu}(\text { had } 2)=4.9(.5) \times 10^{-10} .
$$

Doubts on the reliability of the accuracy of the hadronic light-by-light scattering contribution above have been recently raised in refs. [4] and [5]. As emphasized by Martin Einhorn 
[5], the issue is important for the interpretation of the new experiment and crucial for drawing inferences about potential new physics. The purpose of this note is to analyze the hadronic contributions to $a_{\mu}$ from the point of view of low energy QCD and to show that, within this framework, it might be possible to improve the determination of $a_{\mu}($ had 2$)$ to a sufficiently safe accuracy for a useful interpretation of the new BNL-experiment.

1. I suggest we look first at the well known contribution from hadronic vacuum polarization in Fig. 1a which we shall denote $a_{\mu}($ had $1 a)$. It is usually expressed in the form of an integral representation $\left(e^{2}=4 \pi \alpha, \hbar=c=1\right)$

$$
a_{\mu}(\text { had } 1 a)=\frac{\alpha}{\pi} \int_{0}^{\infty} \frac{d t}{t} K\left(t / m_{\mu}^{2}\right) e^{2} \frac{1}{\pi} \operatorname{Im} \Pi^{(H)}(t)
$$

which is a convolution of the hadronic spectral function $\frac{1}{\pi} \operatorname{Im} \Pi^{(H)}(t)$, related to the total $e^{+} e^{-} \rightarrow$ hadrons cross-section $\sigma(t)\left(m_{e} \rightarrow 0\right)$ by

$$
\sigma(t)=\frac{4 \pi^{2} \alpha}{t} e^{2} \frac{1}{\pi} \operatorname{Im} \Pi^{(H)}(t)
$$

with the QED function

$$
K\left(t / m_{\mu}^{2}\right)=\int_{0}^{1} d x \frac{x^{2}(1-x)}{x^{2}+(1-x) t / m_{\mu}^{2}},
$$

which is positive and monotonically decreasing from $t=0$ to $t=\infty$. I propose we try to calculate $a_{\mu}($ had $1 a)$, without using the experimentally known cross-section $\sigma(t)$. This calculation will then serve us as a test of how well we understand - theoretically - $a_{\mu}($ had $1 a)$. (If here we fail to reproduce the phenomenological determination, there is little hope to expect that we can make a reliable estimate of $a_{\mu}($ had 2).)

For our purposes, it is also convenient to express $a_{\mu}(\mathrm{had} 1 a)$ using the integral representation $[6]$

$$
a_{\mu}(h a d 1 a)=\frac{\alpha}{\pi} \int_{0}^{1} d x(1-x)\left[-e^{2} \Pi_{R}^{(H)}\left(\frac{x^{2}}{1-x} m_{\mu}^{2}\right)\right]
$$

where $\Pi_{R}^{(H)}\left(Q^{2}\right)$ denotes the renormalized $\left(\Pi_{R}^{(H)}(0)=0\right)$ hadronic photon self-energy. The link between the two integral representations in (6) and (9) is provided by the once subtracted dispersion relation $\left(Q^{2} \equiv-q^{2} \geq 0\right.$ in our metric,,,+---$)$

$$
\Pi_{R}^{(H)}\left(Q^{2}\right)=\int_{0}^{\infty} \frac{d t}{t} \frac{q^{2}}{t-q^{2}} \frac{1}{\pi} \operatorname{Im} \Pi^{(H)}(t) .
$$


The bulk of the integral in (9) is governed by the low energy behaviour of $\Pi_{R}^{(H)}\left(Q^{2}\right)$. The typical momemtum of the virtual photon in Fig. 1a is $Q^{2} \sim m_{\mu}^{2}$. These are momenta values much smaller than the characteristic scale $\Lambda_{\chi}$ of the spontaneous chiral symmetry breaking in QCD with three light flavours $\left(\Lambda_{\chi} \simeq 1 G e V\right)$. Therefore, the appropriate way to look at this problem is within the framework of the low energy effective field theory of QCD i.e., chiral perturbation theory $(\chi \mathrm{PT})$.

Since $\Pi_{R}^{(H)}(0) \equiv 0$, the leading contribution to $a_{\mu}($ had $1 a)$ from tree level couplings in $\chi \mathrm{PT}$ comes from the $O\left(p^{6}\right)$ effective Lagrangian ; and more precisely from the term proportional to $F_{\mu \nu} \square F^{\mu \nu}$. The coupling constant of this term is related to the slope at the origin of the hadronic photon self-energy. The relevant effective Lagrangian we are concerned with here is

$$
\mathcal{L}_{e f f}=-\frac{1}{4}\left\{F_{\mu \nu} F^{\mu \nu}-P_{1} e^{2} \partial^{\lambda} F^{\mu \nu} \partial_{\lambda} F_{\mu \nu}+\ldots\right\}
$$

where

$$
P_{1}=-\left.\frac{\partial \Pi_{R}^{(H)}\left(Q^{2}\right)}{\partial Q^{2}}\right|_{Q^{2}=0}
$$

In QCD, and in the large $N_{c}$ (number of colours) approximation [7], the constant $P_{1}$ adquires a leading contribution of $O\left(N_{c}\right)$. We can then conclude that to leading order in the $1 / N_{c^{-}}$ expansion of QCD and in the chiral limit, to lowest non-trivial order in $\chi \mathrm{PT}$, the calculation of $a_{\mu}($ had $1 a)$ can be reduced to the determination of only one coupling constant of the $O\left(p^{6}\right)$ chiral effective Lagrangian of QCD, with the result [8]

$$
a_{\mu}(\text { had } 1 a) \simeq\left(\frac{\alpha}{\pi}\right)^{2} m_{\mu}^{2} \frac{4 \pi^{2}}{3} P_{1} .
$$

What do we know about the constant $P_{1}$ ? As already shown, $P_{1}$ is an $O\left(p^{6}\right)$ coupling and, unfortunately, our present knowledge in $\chi \mathrm{PT}$ stops at $O\left(p^{4}\right)$. The coupling constants of the $O\left(p^{4}\right)$ effective chiral Lagrangian of QCD have been determined phenomenologically in a series of beautiful papers by Gasser and Leutwyler [9]. It has subsequently been shown [10, 11] that these constants are practically saturated by the lowest resonance exchanges and particularly by vector-exchange whenever vectors can contribute. We can adopt this phenomenological observation to obtain an estimate of $P_{1}$. Using the most natural description of vector mesons in terms of vector fields as discussed e.g. in ref. [12], one obtains the result

$$
P_{1}=\frac{4}{3} \frac{f_{V}^{2}}{M_{V}^{2}}
$$


where $f_{V}$ denotes the coupling of vector mesons to the electromagnetic field $\left(f_{V} \simeq 0.2\right)$ and $M_{V}$ is the vector meson mass $\left(M_{V} \simeq 770 M e V\right)$. The resulting estimate of $a_{\mu}($ had $1 a)$ in eq. (13) is then

$$
a_{\mu}[\text { had } 1 a, \text { eqs. }(13,14)] \simeq 7.1 \times 10^{-8},
$$

to be compared to the latest phenomenological determinations $[3,13,14]$

$$
a_{\mu}(\operatorname{had} 1 a)= \begin{cases}7.068(0.059)(0.164) \times 10^{-8}, & (a) \\ 6.84(0.11) \times 10^{-8}, & (b) \\ 7.100(0.105)(0.49) \times 10^{-8} & (c)\end{cases}
$$

where in (16a) and (16c) the first error is statistical and the second systematic. The agreement of our $\chi \mathrm{PT}$ estimate with the full phenomenological determination is embarrassingly good.

At this point some readers will object that the estimate in (15) uses, after all, phenomenological input - the ratio $f_{V}^{2} / M_{V}^{2}$. I want to discuss next, as a possible alternative, how well the extended Nambu Jona-Lasinio model (ENJL-model) of low energy QCD, which has recently been developped in refs. [15] and [16], does in calculating $a_{\mu}($ had 1a). The ENJL-model can be viewed as an approximation of large- $N_{c}$ QCD, where the only new interaction terms retained after integration of the high frequency modes of the quark and gluon fields down to a scale $\Lambda_{\chi}$ at which spontaneous chiral symmetry breaking occurs, are those which can be cast in the form of four-fermion operators. The parameters of the model are then $\Lambda_{\chi}$ and the two coupling constants $G_{s}$ and $G_{v}$ of the two-possible (scalar-pseudoscalar) and (vector-axial) four-fermion couplings. These two couplings can be traded for the mass $M_{Q}$ of the constituent chiral quark, which appears as a non-trivial solution to the gap equation involving $G_{s}$; and the effective axial coupling $g_{A}$ of the constituent chiral quarks to the pseudoscalar Goldstone bosons [17]

$$
g_{A}=\frac{1}{1+4 G_{v} \frac{M_{Q}^{2}}{\Lambda_{\chi}^{2}} \Gamma\left(0, \frac{M_{Q}^{2}}{\Lambda_{\chi}^{2}}\right)},
$$

where $\Gamma(0, \varepsilon)$ denotes the incomplete gamma function

$$
\Gamma(n, \varepsilon)=\int_{\varepsilon}^{\infty} \frac{d z}{z} e^{-z} z^{n} .
$$

In ref. [16] the QCD vector two-point function, within the approximation provided by the ENJL-model, has been calculated to all orders in powers of momenta at the leading order in the $1 / N_{c}$-expansion with the result

$$
\Pi_{V}^{(1)}=\frac{\bar{\Pi}_{V}^{(1)}\left(Q^{2}\right)}{1+Q^{2} \frac{8 \pi^{2} G_{v}}{N_{c} \Lambda_{\chi}^{2}} \bar{\Pi}_{V}^{(1)}\left(Q^{2}\right)},
$$


where

$$
\bar{\Pi}_{V}^{(1)}\left(Q^{2}\right)=\frac{N_{c}}{16 \pi^{2}} 8 \int_{0}^{1} d y y(1-y) \Gamma\left(0, \frac{M_{Q}^{2}+Q^{2} y(1-y)}{\Lambda_{\chi}^{2}}\right) .
$$

In terms of $\Pi_{V}^{(1)}$, the hadronic photon self-energy is given by the expression $\left(\Sigma Q_{i}^{2}=2 / 3, i=\right.$ $u, d, s)$

$$
\Pi_{R}^{(H)}\left(Q^{2}\right)=\frac{2}{3}\left(\Pi_{V}^{(1)}\left(Q^{2}\right)-\Pi_{V}^{(1)}(0)\right)
$$

and the $O\left(p^{6}\right)$ coupling constant $P_{1}$ defined in eqs. (11) and (12) can then be easily obtained:

$$
P_{1}(\mathrm{ENJL})=\frac{N_{c}}{16 \pi^{2}} \frac{2}{3} \frac{1}{M_{Q}^{2}} \frac{4}{15}\left[\Gamma\left(1, \frac{M_{Q}^{2}}{\Lambda_{\pi}^{2}}\right)+\frac{5}{4} \frac{1-g_{A}}{g_{A}}\right] .
$$

In the limit $G_{v} \Rightarrow 0$ (i.e., $g_{A}=1$ ) and $\Lambda_{\chi}^{2}>>M_{Q}^{2}$ we recover the result of the constituent chiral quark model [18], [19]. Using the input parameter values

$$
M_{Q}=265 M e V, \Lambda_{\chi}=1165 M e V, g_{A}=0.61
$$

corresponding to the fit 1 in ref. [15] one gets

$$
a_{\mu}[\operatorname{had} 1 a ; \text { eqs. }(13,22)]=6.7 \times 10^{-8}
$$

while, using the same $M_{Q}=265 \mathrm{MeV}$ value, the constituent quark model prediction comes out to be rather low $\left(3.9 \times 10^{-8}\right)$. We conclude that the ENJL-model does well in reproducing the semiphenomenological estimate of eq. (15).

The deep reason why I am discussing the ENJL-model is that, as I shall next show, it offers the possibility to go beyond the leading $O\left(p^{6}\right)$ contribution of $\chi \mathrm{PT}$. When, later, we discuss the hadronic light-by-light contribution, this will appear to be a crucial issue.

In trying to estimate the $O\left(p^{8}\right)$ correction from the hadronic photon self-energy to the integral in eq. (9) there appears an interesting difficulty. Once we factorize the second derivative at the origin of the photon self-energy in eq. (9), the remaining integral over the $x$-variable diverges logarithmically in the ultraviolet region $(x \rightarrow 1)$. In the language of $\chi \mathrm{PT}$ this means that a genuine local counterterm of the type $\bar{\psi} \sigma^{\mu \nu} \psi F_{\mu \nu}$ has to be introduced, with a constant which after renormalization will absorb the $U V$-divergence of the previous loop integral. However, the finite part of this new constant is not fixed by arguments of symmetry and depends on the details of the dynamics of the underlying theory. It is precisely in this sense that the ENJL-model helps, without appealing to extra phenomenological input. If we 
insert the full expression for the hadronic photon self-energy given by eqs. $(21,19,20)$ in the integrand of eq.(9), the integral over the $x$-variable is convergent. Strictly speaking, the $Q^{2}$ dependence of the hadronic photon self-energy predicted by the ENJL-model is expected to be adequate up to $Q^{2}$ values smaller than $\Lambda_{\chi}^{2}$. Beyond that we enter a regime where a description in terms of perturbative QCD is certainly more appropriate. We have here another observable which, like the $\pi^{+}-\pi^{0}$ electromagnetic mass difference recently discussed in ref. [16] (see also references therein), offers the possibility to test the quality of the matching between longdistance behaviour and short-distance behaviour. I don't want to discuss this in detail now, because it would take us too far away from the main purpose of this note. Here, I shall only give the result I get for the total $a_{\mu}($ had $1 a)$ using the ENJL-model expression of $\Pi_{R}^{(H)}\left(Q^{2}\right)$ for the evaluation of the long-distance part contribution; and using perturbative QCD for the calculation of the short-distance part contribution. The matching between short-and long-distances is defined by the optimal choice of an $\widehat{x}$ in the integral (9) which minimizes the variation of the total long $\left(\int_{0}^{\widehat{x}}\right)$ - plus short $\left(\int_{\widehat{x}}^{1}\right)$ - distance contribution. With the same input parameter values as in eq.(23), I find

$$
a_{\mu}(\operatorname{had} 1 a, \text { ENJL })=6.7 \times 10^{-8} .
$$

This is the result corresponding to the optimal choice $\widehat{x} \simeq 0.9$. The stability around this value is very good. (Perhaps I should clarify that, in terms of the $t$-variable in the hadronic spectral function representation of eqs. $(6,7,8)$, the value $\widehat{x} \simeq 0.9$ corresponds to an "equivalent" $\widehat{t} \simeq 2 G e V^{2}$; which is very reasonable). The fact that the two results in (24) and (25) are exactly the same is a numerical coincidence. If instead of using the values of the input parameters in (23), I use the values corresponding to fit 2 in [15] (i.e., $M_{Q}=263 \mathrm{MeV}, \Lambda_{\chi}=$ $\left.1048 \mathrm{MeV}, g_{A}=0.62\right)$ the result is $a_{\mu}(\mathrm{had} 1 \mathrm{a}$, ENJL fit 2$)=6.6 \times 10^{-8}$.

So far, I have not discussed the explicit contributions to $a_{\mu}\left(\right.$ had $1 a$ ) from $\pi^{+} \pi^{-}$(and $K^{+} K^{-}$) vacuum polarization insertions in Fig.1a. In the framework of $\chi \mathrm{PT}$, these contributions appear as chiral loops induced by the lowest order effective Lagrangian

$$
\mathcal{L}_{e f f}=\frac{1}{4} f_{\pi}^{2} \operatorname{tr} D_{\mu} U D^{\mu} U^{\dagger}+\ldots
$$

where $U$ is a unitary $3 \times 3$ matrix with det $U=1$ which collects the pseudoscalar fields and $D_{\mu} U$ the covariant derivative

$$
D_{\mu} U=\partial_{\mu} U-i\left(v_{\mu}+a_{\mu}\right) U+i U\left(v_{\mu}-a_{\mu}\right)
$$


with $v_{\mu}\left(a_{\mu}\right)$ external vector (axial) field sources. In our case : $v_{\mu}=e Q A_{\mu}$ and $a_{\mu}=0$, where $Q=1 / 3 \operatorname{diag}(2,-1,-1)$ and $A_{\mu}$ is the electromagnetic gauge-field. The Lagrangian in (26) has an effective coupling

$$
-i e A_{\mu}\left(\pi^{+} \stackrel{\leftrightarrow}{\partial_{\mu}} \pi^{-}+K^{+} \overleftrightarrow{\partial_{\mu}} K^{-}\right)
$$

which leads to the lowest order (i.e., $\left.O\left(p^{4}\right)\right)$ hadronic spectral function in $\chi \mathrm{PT}$ :

$$
\frac{1}{\pi} \operatorname{Im} \Pi_{\chi l o o p}^{(H)}(t)=\frac{1}{16 \pi^{2}} \frac{1}{3}\left(1-\frac{4 m_{\pi}^{2}}{t}\right)^{3 / 2} \Theta\left(t-4 m_{\pi}^{2}\right)+\pi \leftrightarrow K
$$

There are two important features emerging here. First, the fact that, by contrast to the previous contributions we have discussed, this spectral function is non-leading in the $1 / N_{c^{-}}$ expansion. (This is in fact a rather general property of chiral loops in QCD.) Second, the observation that when $\frac{1}{\pi} \operatorname{Im} \Pi_{\chi l o o p}^{(H)}$ is inserted in the integral representation in eq.(6), although nominally it is a leading $O\left(p^{4}\right)$ term in chiral power counting, it contributes a very small amount to $a_{\mu}($ had $1 a)$. Numerically, I find $\left(\pi^{+} \pi^{-}+K^{+} K^{-}\right)$

$$
a_{\mu}(\operatorname{had} 1 ; \chi \text { loops })=(0.71+0.07) \times 10^{-8}
$$

This small result is essentially due to the empirical fact that the physical threshold for $\pi^{+} \pi^{-}$ (and $K^{+} K^{-}$) production is much bigger than the muon mass, therefore suppressing further the, already $1 / N_{c^{-}}$suppressed, $O\left(p^{4}\right)$ chiral loop contribution. More quantitatively, for $t \geq 4 m_{\pi}^{2}$ the function $K(t)$ in eq.(8) is very well approximated by its assymptotic $\frac{1}{3} m_{\mu}^{2} / t$ - behaviour. Therefore, the contribution from chiral loops is practically dominated by the slope of their contribution to the photon self-energy. The slope in question is

$$
P_{1}(\chi l o o p)=\frac{1}{16 \pi^{2}} \frac{1}{30}\left(\frac{1}{m_{\pi}^{2}}+\frac{1}{M_{k}^{2}}\right)
$$

about one order of magnitude smaller than the leading $O\left(N_{c}\right)$ slope values in eqs.(14) and (22). The result in eq.(30) is the leading chiral loop correction, and as such has to be added to our full estimate in the chiral limit of the $O\left(N_{c}\right)$ contribution in eq.(25). This sum gives the best theoretical estimate I can think of at present for $a_{\mu}($ had 1a) - without using the empirical knowledge of the $e^{+} e^{-} \rightarrow$ hadrons total cross-section - i.e.,

$$
a_{\mu}(\operatorname{had} 1 a, \text { Theory })=7.5 \times 10^{-8}
$$


It is in the high ball park of the phenomenological determinations in $(16 a, b, c)$, but not bad at all. We conclude that we understand reasonably well, within the framework of low energy $\mathrm{QCD}, a_{\mu}(\operatorname{had} 1 a)$.

2. It is time to examine now the contribution from the hadronic light-by-light scattering in Fig.2 i.e. $a_{\mu}\left(\right.$ had 2). Again, the typical momenta of the virtual photons in Fig.2 is $Q^{2} \sim m_{\mu}^{2}$; and the appropriate framework to analyze the problem is the one of $\chi \mathrm{PT}$. At first sight, the leading contribution to $a_{\mu}($ had 2) from direct tree level couplings appears to come from the $O\left(p^{8}\right)$ chiral effective Lagrangian with four electromagnetic field strength tensors i.e., the Lagrangian $\left(\Sigma Q_{i}^{4}=2 / 9, \quad i=u, d, s\right)$

$$
\begin{aligned}
\mathcal{L}_{e f f}= & \frac{N_{c}}{16 \pi^{2}} \frac{1}{360} \frac{1}{M_{Q}^{4}} \frac{2}{9} e^{4}\left\{4 h_{1}\left(F_{\mu \nu}(x) F^{\mu \nu}(x)\right)^{2}\right. \\
& \left.+7 h_{2}\left(\varepsilon^{\mu \nu \rho \sigma} F_{\mu \nu}(x) F_{\rho \sigma}(x)\right)^{2}\right\} .
\end{aligned}
$$

This is the QCD-analogue of the famous Euler-Heisenberg effective Lagrangian of QED. [20]. (For an excellent book on the subject see ref. [21].) With the normalization I have chosen, the coupling constants $h_{1}$ and $h_{2}$ are dimensionless. In the constituent chiral quark model $[18,19,15] h_{1}=h_{2}=1$. In QCD these effective couplings appear to leading $O\left(N_{c}\right)$ in the $1 / N_{c^{-}}$ expansion. (This is why I have already pull out the $N_{c}$-factor in (33).) The direct insertion in the muon vertex diagram of Fig. 2 of the local light-by-light scattering couplings given by the effective Lagrangian in (33) leads however to $U V$-divergent integrals. The situation here is rather similar to the one we already encountered in the case of hadronic vacuum polarization when one tries to insert the local $O\left(p^{8}\right)$ photon self-energy coupling. Again, what we find now is that we need more information about the underlying QCD dynamics than just the two local couplings $h_{1}$ and $h_{2}$. In view of its phenomenological successes it seems reasonable to expect that the ENJL-model we discussed earlier may provide the required help. More on that later. First, let us examine other possible contributions to $a_{\mu}($ had 2$)$ within the framework of $\chi \mathrm{PT}$.

There is of course the contribution from the hadronic light-by-light scattering amplitude induced by the effective lowest order coupling in (26). Like in the case of the hadronic vacuum polarization, it gives rise to an $O\left(p^{4}\right)$ contribution, which is also nonleading in the $1 / N_{c}$-expansion. The chiral loop calculation of the light-by-light scattering tensor $\Pi^{\mu \alpha \beta \gamma}\left(k, q_{1}, q_{2}, q_{3}\right)$ leads to a rather complicated expression. In order to get some 
feeling, we can however consider the limit where all the photon momenta are negligible with respect to the physical pion mass. This limit is described by the Euler-Heisenberg effective Lagrangian of scalar-QED [21] i.e.,

$$
\begin{aligned}
& \mathcal{L}_{\text {eff }}(\text { scalar-QED })=-\frac{1}{4} F_{\mu \nu} F^{\mu \nu}+\frac{1}{16 \pi^{2}} \frac{1}{30} \frac{1}{m_{\pi}^{2}} e^{2} \partial^{\lambda} F^{\mu \nu} \partial_{\lambda} F_{\mu \nu} \\
& +\frac{1}{16 \pi^{2}} \frac{1}{1440} \frac{1}{m_{\pi}^{4}} e^{4}\left\{7\left(F_{\mu \nu} F^{\mu \nu}\right)^{2}+\left(\varepsilon^{\mu \nu \rho \sigma} F_{\mu \nu} F_{\rho \sigma}\right)^{2}\right\}+\ldots
\end{aligned}
$$

As already discussed earlier, the second term in the r.h.s. above governs the slope of the photon self-energy induced by the lowest order chiral loop (see eq.(31)). The third term is the resulting one for the effective light-by-light scattering. From the comparison between (33) and (34) one can see that for values $h_{1} \simeq h_{2} \simeq O(1)$, the order of magnitude of the direct $O\left(N_{c}\right)$ couplings are about the same size (or smaller for the $(F F)^{2}-$ term) than those induced by the pion chiral loop. The moral here is that the contribution to $a_{\mu}($ had 2$)$ from chiral loops, although $1 / N_{c}$-suppressed, may well give rise to a sizeable contribution.

There is yet another contribution to hadronic light-by-light scattering which in $\chi \mathrm{PT}$ appears as a tree level contribution to $O\left(p^{6}\right)$ in the chiral expansion; but contrary to the $O\left(p^{4}\right)$ contribution which we have just discussed, it is leading in the $1 / N_{c}$ - expansion. It is the one induced by the Adler [22], Bell and Jackiw [23] anomaly which in $\chi \mathrm{PT}$ is incorporated in the so called Wess-Zumino-Witten term (WZW) [24,25]. When restricted to two external photons and one pseudoscalar field, the WZW-term has the simple form

$$
\mathcal{L}_{A B J}=\frac{N_{c}}{16 \pi^{2}} \frac{1}{2} e^{2} \epsilon^{\mu \nu \rho \sigma} F_{\mu \nu}(x) F_{\rho \sigma}(x) \frac{1}{f_{\pi}}\left(\pi^{0}(x)+\frac{1}{\sqrt{3}} \eta(x)\right) .
$$

The $\pi^{0}(\eta)$ propagator between two effective ABJ-vertices brings down the resulting $O\left(p^{4}\right) \times$ $O\left(p^{4}\right)$ light-by-light scattering amplitude to $O\left(p^{6}\right)$; and because of the factor $1 / f_{\pi}^{2}$ the overall contribution is $O\left(N_{c}\right)$, and therefore leading in the $1 / N_{c^{-}}$expansion. Obviously, this contribution cannot be neglected; and in fact, the simple power counting arguments above tell us that it could be the dominant contribution.

The previous analysis of $a_{\mu}($ had 2$)$ within the framework of $\chi \mathrm{PT}$ has served to identify three distinct sources of a priori important contributions. We want now to discuss their possible evaluation.

The contribution to $a_{\mu}$ (had 2) from the lowest order chiral pion loop can be calculated unambiguously. It has in fact been evaluated numerically by KNO using two different meth- 
ods, with the average result

$$
a_{\mu}(\text { had } 2, \chi \text { pion loop })=-5.12(0.35) \times 10^{-10}
$$

To this result one should add the corresponding contribution from the chiral kaon loop; presumably rather small.

A straightforward calculation of the contribution to $a_{\mu}($ had 2$)$ from the $O\left(p^{6}\right)$ lightby-light scattering amplitude resulting from the $\pi^{0}(\eta)$-exchange between two ABJ-vertices leads to $U V$-divergent integrals. Once again, this is an indication that more about the underlying QCD dynamics than just the local $\pi^{0}(\eta) \gamma \gamma O\left(p^{4}\right)$ coupling is needed. KNO, in their evaluation of the " $\pi^{0}$-exchange" contribution, have solved the problem by using a vector-meson-dominance (VMD) inspired regularization of the three photon-propagators; i.e., they use the prescription $\left.Q_{i}^{2} \equiv-q_{i}^{2} \quad i=1,2,3\right)$ :

$$
\frac{-1}{Q_{i}^{2}} \rightarrow \frac{-1}{Q_{i}^{2}}+\frac{1}{Q_{i}^{2}+M_{V}^{2}}
$$

It turns out that this VMD - prescription is in fact very close to the result one obtains in the ENJL-model [16], after summing all orders in powers of momenta at the leading order in the $1 / N_{c}$ - expansion, which leads to the effective replacement :

$$
\frac{-1}{Q_{i}^{2}} \rightarrow \frac{-1}{Q_{i}^{2}} \frac{1}{1+Q_{i}^{2} \frac{8 \pi^{2} G_{v}}{N_{c} \Lambda_{\chi}^{2}} \bar{\Pi}_{V}^{(1)}\left(Q_{i}^{2}\right)}=\frac{-M_{V}^{2}\left(Q_{i}^{2}\right)}{Q_{i}^{2}\left[Q_{i}^{2}+M_{V}^{2}\left(Q_{i}^{2}\right)\right]}
$$

with $\bar{\Pi}_{V}^{(1)}\left(Q^{2}\right)$ as given in eq.(20), and $M_{V}\left(Q^{2}=0\right)=M_{V}$. Numerically, the result found by KNO from the " $\pi^{0}$-exchange" term, and using the VMD-prescription in (37), is

$$
a_{\mu}\left(\pi^{0}\right)=6.5(0.6) 10^{-10}
$$

The corresponding contribution from $\eta$-exchange which should be added to (39) is likely to be negligible.

An improved calculation of the " $\pi^{0}$-exchange" contribution within the framework of the ENJL-model is possible. Schematically, it corresponds to the replacement of the $\pi^{0}$-exchange tree level diagram in Fig. 3a by the one in Fig. 3b. The round dots (o) in this figure represent four-fermion vector like couplings; the square dots ( $\square$ ) four-fermion axial-vector like couplings. The horizontal string of bubbles in Fig. 3b has already been calculated (see 
section 3.2 of ref. [16]). It contains in particular the pion (eta) - pole(s), but also axial-vector contributions. The three-point functions at the vertices of Fig. 3b; i.e., the links $\times \bigcirc_{\circ}^{\square}$ and $\square \bigcirc_{\circ}^{\circ}$, have non-trivial $Q_{i}^{2}$ - dependence. In KNO's calculation, they have been approximated by the corresponding local ABJ-vertices in (35).

Finally we turn our attention back to the leading $O\left(N_{c}\right)$ contribution we first discussed, which in $\chi \mathrm{PT}$ starts at $O\left(p^{8}\right)$. This is an independent contribution which has to be evaluated separately; and, contrary to what has been done so far in the literature, it should be added to the two other contributions previously discussed. KNO's numerical evaluation of what they call the "quark-loop" contribution provides in my opinion a good first approximation to the required calculation. The constituent quark-loop graphs of refs. [3] and [26] correspond to the mean field approximation of the ENJL-model of refs. [15,16]. In the case of hadronic vacuum polarization, this is the approximation where $\Pi_{V}^{(1)} \rightarrow \bar{\Pi}_{V}^{(1)}$ (see eqs. (19) and (20)). The result found by KNO for the "quark-loop" contribution is

$$
a_{\mu}(\text { had } 2, \text { quark-loop })=6.0(0.4) \times 10^{-10} .
$$

It corresponds to the choice $M_{u}=M_{d}=300 \mathrm{MeV}$ and $M_{s}=500 \mathrm{MeV}$. (There is also a small contribution from the charm quark with a mass value $M_{c}=1.5 \mathrm{GeV}$ which has been included in (40).)

Again, it is possible to do an improved calculation of the "quark-loop" type contribution within the framework of the ENJL-model. The suggested improvement is schematically represented in Fig. 4. In practice it amounts to the effective replacement indicated in (38) for each photon-propagator. Since here we are working in the chiral limit the $u, d$ and $s$ constituent quark masses should be taken equal. I would expect a sizeable reduction of the "quark-loop" estimate in (40) by perhaps as much as a factor of two.

The main conclusion which emerges from our analyses is that the three contributions in (36), (39), and (40) correspond to well identified different sources in low-energy QCD. The three contributions should be added in order to get an appropriate estimate of $a_{\mu}($ had 2$)$. So far, only the result from the chiral $\pi$-loop contribution in (36) can be considered to be fully reliable. (In that respect I agree with the analyses of ref. [5].) Both the " $\pi^{0}$-exchange" and the "quark-loop" results in (39) and (40) should be considered as first approximation estimates; specially the "quark-loop" result (40). These estimates can however be substantially refined if one is ready to accept the ENJL-model as a good approximation to low-energy QCD at large- 
$N_{c}$. We see no reason why the ENJL-model should fail here, and the results we have obtained in the case of the hadronic vacuum polarization are very encouraging. The improvement can be done following the suggestions I have sketched above. The implementation of this improvement is quite a formidable task, but it should be relatively easy to incorporate in the already existing computer programs developped by Kinoshita et al. A parallel calculation of the 
$\gamma \gamma \rightarrow \gamma \gamma$ cross-section at low energies in the ENJL-model, and its comparison to future experimental results of DA $\Phi$ NE, could help to check the validity of the model.

\section{ACKNOWLEDGEMENTS}

I am grateful to Hans Bijnens, Gilles Esposito-Farèse, Santi Peris and Toni Pich for their comments and suggestions on the topics discussed in this note. 


\section{REFERENCES :}

[1] J. Bailey et al., Phys. Lett., 68 B, 191 (1977) ; F.J.M. Farley and E. Picasso, The muon g-2 Experiments, Advanced Series on Directions in High Energy Physics - Vol. 7 Quantum Electrodynamics, ed. T. Kinoshita, World Scientific 1990.

[2] T. Kinoshita and W.J. Marciano, Theory of the Muon Anomalous Magnetic Moment, Advanced Series on Directions in High Energy Physics - Vol. 7 Quantum Electrodynamics, ed. T. Kinoshita, World Scientific 1990.

[3] T. Kinoshita, B Nižić and Y. Okamoto, Phys. Rev., D 31, (1985), 2108.

[4] R. Barbieri and E. Remiddi, The Daфne Physics Handbook - Vol. II, p. 301 ; ed. L. Maiani et al., Frascati, INFN 1992.

[5] M.B. Einhorn, On the Hadronic Contribution to Light-by-Light Scattering in $g-2$; Preprint UM - TH - 93 - 18, (to be published in Phys. Rev. Comments).

[6] B.E. Lautrup and E. de Rafael, Nuovo Cim. 64 A (1969) 322 ; B.E. Lautrup, A. Peterman and E. de Rafael, Phys. Rep. - Vol. 3 C, No 4 (1972), 193-260.

[7] G. 't Hooft, Nucl. Phys., B 72, (1974), 461.

[8] J.S. Bell and E. de Rafael, Nucl. Phys., B 11, (1969), 611.

[9] J. Gasser and H. Leutwyler, Ann. of Phys. (N.Y.) 158, (1984) 142 ; Nucl. Phys. B 250, (1985), 465, 517, 539 .

[10] G. Ecker, J. Gasser, A. Pich and E. de Rafael, Nucl. Phys. B 321, (1989), 311.

[11] J.F. Donoghue, C. Ramirez, and G. Valencia, Phys. Rev. D 39, (1989), 1947.

[12] G. Ecker, J. Gasser, H. Leutyler, A. Pich, and E. de Rafael, Phys. Lett. B 223, (1989), 425.

[13] J.A. Casas, C. Lopez and F.J. Ynduráin, Phys. Rev. D 32, (1985), 736.

[14] L.M. Kurdadze et al., Yad. Fiz. 40, (1984), 451 ; Sov. J. Nucl. Phys. 40, (1984), 286.

[15] J. Bijnens, Ch. Bruno and E. de Rafael, Nucl. Phys. B 390, (1993), 501.

[16] J. Bijnens, E. de Rafael and H. Zheng, Low-Energy Behaviour of Two-Point Functions of Quark Currents ; Preprint CERN - TH. 6924 / 93, CPT - 93 / P.2917, Nordita - 93 / 43 N, P.

[17] S. Peris and E. de Rafael, Phys. Lett. B 309, (1993), 389.

[18] A. Manohar and H. Georgi, Nucl. Phys. B 234, (1984), 1189.

[19] D. Espriu, E. de Rafael and J. Taron, Nucl. Phys. B 345, (1990), 22 ; (Erratum B 355, 
(1991), 278).

[20] E. Euler, Ann. Phys. (Leipzig) 26, (1936), 398 ; E. Euler and W. Heisenberg, Z. Phys. 98, (1936), 714 .

[21] J. Schwinger, Particles, Sources and Fields - Vol II, Addision-Wesley Pub. Company (1973).

[22] S.L. Adler, Phys. Rev. 177, (1969), 2426.

[23] J.S. Bell and R. Jackiw, Nuovo Cim. 60 A, (1969), 47.

[24] J. Wess and B. Zumino, Phys. Lett. 37 B, (1971), 95.

[25] E. Witten, Nucl. Phys. B 223, (1983), 422.

[26] J. Calmet, P. Narison, M. Perrotet and E. de Rafael, Phys. Lett. 16 B, (1976), 283 ; Rev. Mod. Phys. 49, (1977), 21. 


\section{FIGURE CAPTIONS :}

Fig. 1 Hadronic vacuum polarization to the second order muon $g-2$ (a) ; and to the fourthorder muon $g-2$ (b).

Fig. 2 Hadronic light-by-light scattering contribution to the muon $g-2$.

Fig. 3 Hadronic light-by-light scattering contribution induced by the combination of two three-point functions. Graph (a) represents the $\pi^{\circ}$-exchange contribution. Graph (b) the corresponding generalized contribution within the ENJL-model. The square dots (口) represent four-fermion axial-vector like couplings ; the round dots (०) fourfermion vector like couplings.

Fig. 4 Hadronic light-by-light scattering contribution induced by the irreducible four-point function. Graph (a) represents the mean field approximation. Graph (b) the full ENJL-model contribution to all orders in momenta for the off-shell photons. 
This figure "fig1-1.png" is available in "png" format from: http://arxiv.org/ps/hep-ph/9311316v1 
This figure "fig1-2.png" is available in "png" format from: http://arxiv.org/ps/hep-ph/9311316v1 